\title{
Effets de la mise en œuvre de la classe inversée à travers Edmodo dans l'apprentissage de la grammaire
}

\author{
${ }^{1}$ Dante DARMAWANGSA \& ${ }^{2}$ Ariessa RACMADHANY \\ 1,2 Département de français, Universitas Pendidikan Indonesia - Indonésie
}

Reçu le 4 août 2018 I Accepté le 22 décembre 2018

\begin{abstract}
RÉSUMÉ. La stratégie de classe inversée, comme l'une des stratégies de l'apprentissage hybride, a eu un impact positif sur le changement des méthodes d'enseignement traditionnelles, qui passent généralement du temps en classe, en les combinant de manière virtuelle. Cette étude tente d'étudier les effets de la stratégie de classe inversée en utilisant Edmodo dans l'apprentissage de la grammaire française. L'étude est menée en utilisant la méthode quantitative avec le modèle quasi expérimental. Les participants à cette recherche sont les étudiants qui ont étudié la grammaire française du niveau A1 / A2 du CECRL dans une université en Indonésie. Les résultats montrent que cette stratégie contribue positivement à l'amélioration des performances des étudiants en matière de grammaire. Cette étude devrait contribuer à offrir un autre aperçu de la conception et des résultats de l'application du matériel d'apprentissage par le biais de la stratégie de la classe inversée, notamment à travers la plateforme Edmodo.
\end{abstract}

Mots-clés : apprentissage hybride, classe inversée, Edmodo, grammaire.

\begin{abstract}
The strategy of flipped classroom, like one of the blended learning's strategies, had a positive impact in the change of the traditional methods of teaching, which normally spend time in the classroom, by combining them with those in a virtual way. This study attemps to investigate the effects of flipped classroom strategy using Edmodo in the French grammar learning. The study is conducted by using the quantitative method with the model quasi-experimental. The participants of this research is the students who studied French grammar on CEFR level A1/A2 in one university in Indonesia. The results show that this strategy contributes positively to the improvement of the students' performances in grammar subject. This study should contribute in offering another insight into the design and results of the application of learning materials through the flipped classroom's strategy, in particular through Edmodo platform.
\end{abstract}

Keywords: blended learning, flipped classroom, Edmodo, grammar.

auteur correspondant : dante.darmawangsa@upi.edu

Pour citer cet article (Style APA) : Darmawangsa, D. \& Racmadhany, A. (2018). Effets de la mise en œuvre de la classe inversée à travers Edmodo dans l'apprentissage de la grammaire. Francisola : Revue Indonésienne de la langue et la littérature françaises, 3(2), 176-184. doi : 10.17509/francisola.v3i2.15750 


\section{INTRODUCTION}

Dans l'apprentissage des langues, tous les éléments de la langue, à savoir le vocabulaire ou la grammaire, sont importants à apprendre. La précision de l'utilisation du vocabulaire et la structure de la grammaire jouent un rôle important dans la transmission des messages dans la communication. Cependant, l'apprentissage de la grammaire est considéré comme plus difficile que celui du vocabulaire, et la maîtrise de la grammaire est considéré comme le fondement de la maitrise d'une langue (Alhaysony \& Alhaisoni, 2017)

Dans le contexte de l'apprentissage d'une langue étrangère, les apprenants ont souvent des difficultés à maîtriser la grammaire. Pourtant, ce n'est pas seulement les apprenants, même les enseignants ont également des difficultés à enseigner la grammaire (voir Al-Mekhlafi \& Nagaratnam, 2011). Les erreurs rencontrées par l'apprenant peuvent être trouvées à partir des erreurs commises. Nous pouvons voir les cas de ces erreurs dans les résultats des recherches menées par Adewuyi, Bernard, \& Adewuyi (2015); Chachu (2016); et Redhouane \& Gamal, (2017).

Cependant, l'enseignant a également de la difficulté à enseigner la grammaire, notamment du point de vue du temps d'enseignement. Ils n'ont pas assez de temps pour explorer le texte et proposer des activités d'apprentissage (Al-Mekhlafi \& Nagaratnam, 2011). En plus, ils n'ont pas assez de temps pour appliquer différentes techniques ou méthodes d'enseignement et pour bien enseigner tous les aspects grammaticaux (Alhaysony \& Alhaisoni, 2017).

La pénurie de temps est devenu un obstacle non seulement pour les enseignants, mais également pour les apprenants. Avec un temps limité, les étudiants sont devenus moins de matériels à explorer et de faire des exercices. Cela nécessite donc d'une méthode afin de résoudre au problème des contraintes de temps. La méthode d'apprentissage hybride ou l'apprentissage mixte, connu comme «Blended Learning», pourrait être la solution à ce problème. Cette méthode applique les processus d'apprentissage traditionnels sous forme de processus $\mathrm{d}$ 'apprentissage en classe et en ligne (Graham, 2016) En appliquant cette méthode, l'enseignant et les apprenants peuvent explorer les matières et effectuer des activités d'apprentissage qui ne sont pas limitées à la salle de classe.

Les avantages de l'apprentissage hybride ne se limitent pas aux solutions pour résoudre au problème de pénurie de temps, mais ils facilitent également l'interactivité des apprenants (Purnawarman, Susilawati, \& Sundayana, 2016), ainsi que les interactions d'apprenants-d'enseignant (Liu, 2013), réduire, voire éliminer, l'anxiété liée à la communication, incite les élèves à devenir des apprenants (plus) indépendants et autonomes (Kaménova, 2017; Liu, 2013). Avec l'apprentissage hybride, les élèves assistent à son rythme lui donnant plus d'aisance dans son apprentissage (Kaménova, 2017).

L'apprentissage hybride comprend quatre modèles, à savoir (1) Flipped Classroom, (2) Flex, (3) Self-Blended et (4) Enriched Virtual (Beaver, Hallar, Westmaas, \& Englander, 2015). Parmi les quatre modèles, Capone, De Caterina, \& Mazza (2017) soutiennent que l'une des meilleures stratégies pouvant être mise en œuvre pour réaliser un apprentissage hybride est la classe inversée (flipped classroom).

La classe inversée a été développée par Bergman et Sams en 2007 (Warden, 2016). Selon Bergmann \& Sams (2012, p.13), le concept de base de la classe inversée est "ce qui se fait traditionnellement à la maison, et celui qui est traditionnellement effectué comme un devoir est maintenant terminé en classe". Il existe donc des activités d'apprentissage inversées de la classe à la maison. En parlant d'apprentissage, nous parlons donc de taxonomie cognitive appelée taxonomie de Bloom (Alexander, Kratwohl, Bloom, 2001). À ce sujet, dans l'apprentissage de classe inversée, il existe des étapes cognitives qui sont inversées, comme le montre le diagramme suivant de Correa, (2015): 

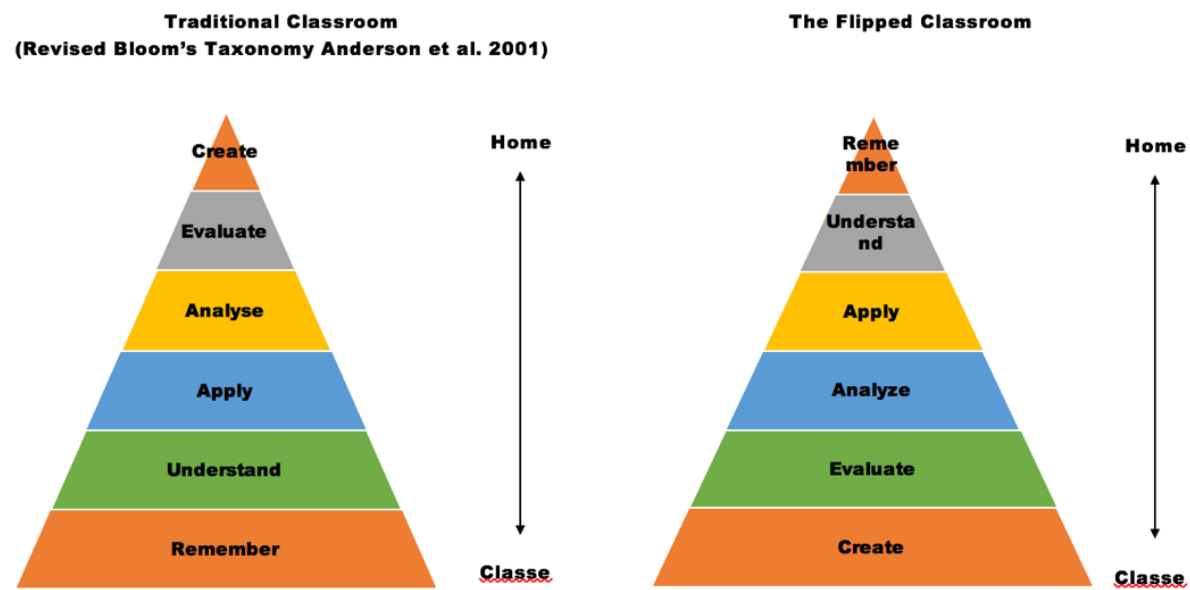

Figure 1. Inversion de la taxonomie de Bloom dans la classe inversée (Correa, 2015, p.121)

En plus, Butt (2014, p.33) dit que «the flipped classroom moving the 'delivery' of material outside of formal class time (through extensive notes, video recorder lectures, and other appropriate means) and using formal class time for student to undertake collaborative and interactive activities relevant to that material » ["la classe inversée consiste à: déplacer ‘le transfert' des matériels en dehors de classes formelles (notes détaillées, conférences sur enregistreur vidéo et autres moyens appropriés), et à utiliser le temps nécessaire pour les activités collaboratives et interactives importantes pour les étudiants."]. Conformément à ce constat, en mettant en œuvre la classe inversée, les apprenants sont demandés à regarder une vidéo, un site ou un article en ligne à la maison avant de venir en classe. Une fois arrivés à l'école, ils discutaient de nouveaux documents sur le Toile par le biais d'activités de coopération, explorant des sujets d'apprentissage fondé sur la recherche (Capone et al., 2017). Cependant, le point essentiel est que la classe inversée est une classe / un environnement où:

1. Students take ownership of the material and use their knowledge to lead one another without prompting from the teacher (a constructivist classroom where students become active learners). [Les apprenants s'approprient le matériel et utilisent leurs connaissances pour se conduire les uns les autres sans se laisser guider par l'enseignant (une classe constructiviste où les élèves deviennent des apprenants actifs).]

2. Interaction and personalized contact time between students and teachers is increased. [Le temps d'interaction et de contact personnalisé entre les étudiants et les enseignants est augmenté.]

3. The teacher is not the sage on the stage, but the guide on the side. [L'enseignant n'est pas le sage sur la scène, mais le guide est sur le côté.]

4. Collaborative work is fluid with students shifting between various simultaneous discussions depending on their needs and interests. [Le travail collaboratif est fluide, les apprenants passant d'une discussion à l'autre en fonction de leurs besoins et de leurs intérêts.]

5. Student-led tutoring and collaborative learning forms spontaneously. [Tutorat dirigé par les étudiants et l'apprentissage collaboratif spontanées.]

6. Students ask exploratory questions and have the freedom to delve beyond core curriculum. [Les apprenants posent des questions exploratoires et ont la liberté d'aller au-delà du programme principal.]

7. Content is permanently archived for review or remediation. [Le contenu est archivé en permanence pour révision ou correction.]

(Correa, 2015)

Les plateformes en ligne pouvant être utilisées dans un environnement d'apprentissage sont variés. Ce qui compte est que l'enseignant peut préparer sur lesquelles les matériels contenant les 
matières et activités d'apprentissage pour que les apprenants puissent y accéder. Quels matériels ? La vidéo est fortement recommandée comme le matériel principal. Pourquoi la vidéo ? Car, il existe une fonctionnalité d'audio qui contient des explications des matières pédagogique, ainsi que des fonctionnalités visuelles ayant pour rôle comme des aides visuelles. Les aides visuelles ne rendent pas seulement l'environnement d'apprentissage amusant afin de stimuler la motivation des apprenants, mais elles peuvent également développer et élargir l'environnement d'apprentissage lorsqu'ils le rencontrent (Shabiralyani, Hasan, Hamad, \& Iqbal, 2015)

Edmodo est une des plateformes existantes qui est capable de soutenir la mise en œuvre de stratégies d'apprentissage de classe inversée. Cette application dispose d'une plate-forme de base du système de gestion de l'apprentissage, connu comme « Learning Management System » (LMS). Grâce à ces plateformes, les enseignants peuvent configurer et gérer facilement leurs cours en ligne: ils peuvent envoyer des questionnaires et des travaux, donner leur avis, recevoir des travaux terminés, attribuer des notes, stocker et partager du contenu dans des fichiers et des liens, gérer un calendrier de cours et en plus, de pouvoir envoyer des notes et des SMS à des étudiants de façons individuels ou à l'ensemble de la classe ; ils peuvent également partager du contenu, soumettre des devoirs, et des quiz, recevoir les commentaires, les notes et les alertes de leur enseignant, ainsi que voter sur sondages (Kara, 2016).

De ce fait, une étude sur la mise en œuvre de stratégie de la classe inversée en utilisant Edmodo est importante et pertinente à effectuer. D'ailleurs, plusieurs études récentes dans le même sujet ont été menées (e.g. Al-Harbi \& Alshumaimeri, 2016; Purnawarman, Susilawati, \& Sundayana, 2016; Warden, 2016; Ekmekci, 2017; Köroğlu \& Çakır, 2017).

Pourtant, ce présent article parle de la mise en place de la stratégie de la classe inversée à travers Edmodo dans l'apprentissage du français langue étrangère (FLE), à savoir la grammaire. Cette étude a été menée auprès du public d'apprenants non francophone afin d'évaluer l'impact de cette stratégie. À ce propos, deux questions de recherches ont été formulés : quels sont les résultats de l'apprentissage de grammaire en mettant en œuvre la stratégie de la classe inversée à travers Edmodo ? Et y a-t-il des différents résultats significatifs entre le groupe expérimental et le groupe de contrôle ? Cette étude vise donc à déterminer si la stratégie de la classe inversée contribue positivement à l'amélioration des performances grammaticales des apprenants de façon significative.

\section{MÉTHODE}

\subsection{Participants}

Les 34 répondants ayant participé à cette étude étaient des étudiants du deuxième semestre du programme d'études de français dans une université en Indonésie, sélectionnés sur la base de la technique d'échantillonnage par choix raisonné (Creswell, 2003). Les personnes choisies sont ceux qui ont un accès Internet à domicile et peuvent utiliser la plateforme Edmodo. Ces répondants étaient répartis en deux classes, à savoir 17 personnes dans la classe expérimentale et 17 personnes dans la classe de contrôle. Ces deux classes reçoivent le même matériel, à savoir du matériel de grammaire française de niveau débutant (A1) du manuel de méthode de français Version Originale 1 , et étudient avec le même enseignant. Dans ce cas, le chercheur agit en tant qu'enseignant à la fois.

\subsection{Méthodologie}

Cette recherche a été menée selon la méthode quasi expérimentale Post-Test-Only Control Group Design. Cette étude utilise des échantillons de deux classes, à savoir la classe expérimentale et la classe de contrôle. Pendant la recherche, les étudiants de la classe de contrôle ont étudié la grammaire française en utilisant des stratégies d'enseignement conventionnelles : face à face en classe et sans support pédagogique. Dans la classe expérimentale, les élèves apprennent la grammaire française dont la stratégie d'apprentissage est la classe inversée à travers la plate-forme Edmodo. 
Pour comparer le niveau de maîtrise entre les classes, un posttest est effectué après la mise en œuvre du traitement de la recherche.

\subsection{Procédures}

Pendant la mise en œuvre du processus de la recherche, le groupe expérimental et le groupe de contrôle étudiaient sept mêmes sujets de la grammaire. Ces sujets étaient l'utilisation du temps passé, des prépositions de lieu, des pronoms d'objet direct, des pronoms d'objet indirect, des pronoms "y", des expressions comparatives et des impératifs. Ces matières sont tirés du manuel de FLE: Version Originale 1. Voici les étapes effectuées dans la classe expérimentale :

- Les enseignants préparaient, choisissaient et traitaient les documents des matières en fonction de sujets basés sur le livre Version Originale 1. De plus, ils préparaient des autres supports en fonction du sujet, à savoir des extraits de la plate-forme Youtube.

- Les enseignants téléchargeaient des documents pédagogiques et des vidéos en fonction des sujets, accompagnés d'instructions pertinentes, y compris des formes de quiz, sur la plate-forme Edmodo cinq jours avant les sessions en face-à-face en classe. Ceci pour que les étudiants puissent les apprendre en dehors des heures des cours. Les étudiants pouvaient soumettre des questions et des commentaires sur chaque téléchargement via la colonne de commentaires sur Edmodo.

- Dans la classe, l'enseignant discutait le contenu des documents et des vidéos téléchargés, notamment des quiz, et des sujets abordés afin d'évaluer le niveau de compréhension des apprenants, et de mettre en commun la perception à la fois sur les matières apprises. Au cours de la discussion, l'enseignant ont également fourni des révisions et exercices nécessaires, soit individuel ou par groupe, avec des rétroactions pertinentes.

Dans la classe de contrôle, l'enseignant n'utilisait pas la stratégie de la classe inversée et n'utilise même pas aucun média. L'enseignant donnait les leçons de manière conventionnelle, à savoir les cours expliquant la grammaire française, suivi d'une discussion, en donnant des exercices, puis en mettant en commun pour donner un retour.

La recherche a été menée pendants 7 séances de cours en fonction du nombre de sujets enseignés. La durée de chaque séance était de 90 minutes.

\subsection{Collecte de données}

\subsubsection{Posttest}

Un posttest a été donné comprenant 50 items de questions qui sont divisés en 5 rubriques. Ces rubriques couvrent tous les sujets des matières de grammaire enseignés. Ce test est conçu pour mesurer l'impact de l'enseignement dispensé (voir pièce jointe).

\subsubsection{Validité et fiabilité}

Pour déterminer la validité des questions, les chercheurs servent à évaluer le test de l'équipe pédagogique qui sont responsable des cours de grammaire $(\mathrm{N}=3)$ afin d'évaluer le niveau de difficulté, la disposition et le nombre de questions proposés. Sur la base de ces considérations, les instruments de test se sont avérés valables car l'équipe d'évaluation a enfin accepté toutes les questions.

D'ailleurs, afin de déterminer la fiabilité du test, la formule 20 de KuderRichardson (simplement appelée KR-20) a été calculée pour chaque question. Sur cette base, le test s'est avéré fiable.

\section{RÉSULTATS ET DISCUSSION}

\subsection{Résultats du posttest}

Les résultats de l'apprentissage de grammaire en mettant en œuvre la stratégie de la classe inversée à travers Edmodo sont reflétés par les résultats du post test du groupe expérimental. Ce test a été donné une semaine après la dernière séance d'expérimentation. Nous distinguons les résultats en trois niveaux de notes: élevée, moyenne, et base. Compte tenu du fait que la note du post test est sur 50, et la note minimum de passation est de 25, nous pouvons donc catégoriser les niveaux de notes comme suivants: du 43 au 50 (note 
élevée), du 34 au 42 (note moyenne), du 25 au 33 (note base).

De ce fait, en analysant les résultats des notes $\mathrm{du}$ posttest, nous pouvons remarquer qu'il y a $29,4 \%$ d'étudiants ayant la note élevée, $41,2 \%$ ayant la note moyenne, et $23,5 \%$ ayant la note base. Pourtant, il est aussi à noter qu'il y a un étudiant (5,9\%) qui n'a pas réussi, car sa note était moins de 25 . Pour les rendre plus clair, nous les présentons sous forme de diagramme suivant :

\section{note élevée $\quad$ note moyenne $\quad$ note base $\quad$ ne pas réussir}

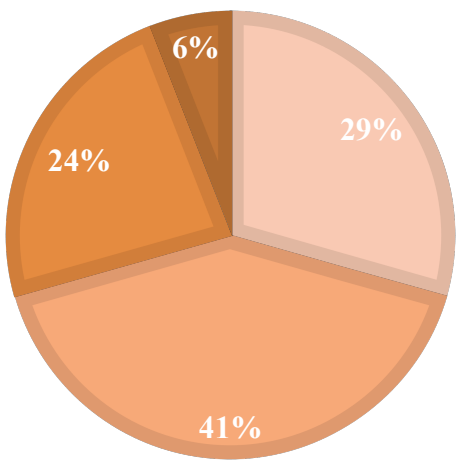

Figure 2. Les résultats des notes du posttest du groupe expérimental

D'ailleurs, par ces résultats, nous ne pouvons pas constater que cette stratégie est efficace ou inefficace dans l'amélioration des connaissances et performances grammaticales des étudiants. Certes, il est nécessaire de faire l'analyse statistique plus profonde dans le cadre de comparer les résultats du posttest du groupe expérimental et ceux du groupe de contrôle. Ceci est dans le cadre de connaître s'il y a des différents résultats significatifs entre ces deux groupes.

\subsection{Analyse statistique}

Tenter à révéler la différence entre le groupe expérimental et de contrôle, nous effectuons la procédure d'analyse de t-test. Les deux groups ont été testés par le même instrument de posttest. Ensuite, l'analyse de t-test pour des échantillons indépendants a été utilisé pour comparer les notes des deux groupes lors du post-test. Un logiciel, appelé SPSS, est utilisé pour analyser la statistique de ces notes. Les résultats sont présentés dans le tableau ci-dessous :

Tableau 1. Analyse descriptive pour le groupe expérimental et le groupe contrôle

\begin{tabular}{|c|c|c|c|c|c|}
\hline Groupe & Nombre & $\begin{array}{c}\text { Note } \\
\text { Moyenne }\end{array}$ & Écart type & Maximum & Minimum \\
\hline groupe de contrôle & 17 & 21,47 & 3,46 & 17 & 30,5 \\
\hline $\begin{array}{c}\text { groupe } \\
\text { expérimental }\end{array}$ & 17 & 36,65 & 6,06 & 22 & 45,5 \\
\hline
\end{tabular}

Basé sur ce tableau ci-dessus, nous pouvons voir que la note moyenne entre ces deux groupes est différente. La note du groupe expérimental est plus élevée $(36,65$ avec l'écart type de 6,06) que celle du groupe de contrôle $(21,47)$ où la note maximum du groupe expérimental est 45,5 tandis que celle du groupe de contrôle est 30,5.

En un coup d'œil, nous pouvons remarquer que la note moyenne entre ces deux groupes a un écart important. Cependant, est-ce que cette différence est significative basée sur la statistique? Afin 
d'y répondre, nous avons effectués un test comparatif. Dans ce cas-là, avant de faire un test comparatif, un test de normalité a été effectué pour déterminer si les données provenaient de la distribution sont normales ou non. Le résultat du test de normalité est présenté dans le tableau suivant:

Tableau 2. Test de normalité

\begin{tabular}{cccc}
\hline \multirow{2}{*}{ Groupe } & \multicolumn{3}{c}{ Test de Normalité } \\
\cline { 2 - 4 } & Statistique & $\mathrm{df}$ & valeur $\mathrm{p}$ \\
\hline Contrôl & 0,927 & 17 & 0,191 \\
Experimental & 0,982 & 17 & 0,974 \\
\hline
\end{tabular}

*) test de Shapiro-Wilk

Valeur p: probability value ( $p$ value)

Les données sont dites normales si la valeur $p$ est supérieure à 0,05 . Basé sur le calcul des données ci-dessus, cela montre que la valeur $p$ pour les deux groupes est supérieure à 0,05. Ainsi, les données des deux groupes possèdent une distribution normale. De ce fait, le test de comparaison utilisé est le Test-t (test-t indépendant) où son résultat est présenté ci-dessous :

Tableau 3. Résultat du Test-T Indépendant

\begin{tabular}{cccccc}
\hline Test & Groupe & Note Moyenne & Écart Type & Test T & sig \\
\hline Post & Groupe Experimental & 21,47 & 3,46 & $-7,78$ & 0,000 \\
Test & Groupe de Contrôle & 36,65 & 6,06 & & \\
\hline
\end{tabular}

Nous pouvons conclure que la note du post test entre les deux groupes a la différence significative si la note sig est inférieure à 0,05. Le calcul précédent montre que la note sig est inferieure 0,05. C'est-à-dire qu'il existe une différence significative entre la performance grammaticale $\mathrm{du}$ groupe expérimental et celle du groupe de contrôle où le groupe expérimental présentait la note moyenne plus élevée par rapport au groupe de contrôle (voir la figure 3).

\section{La note moyenne de groupe experimental et de groupe de controle}

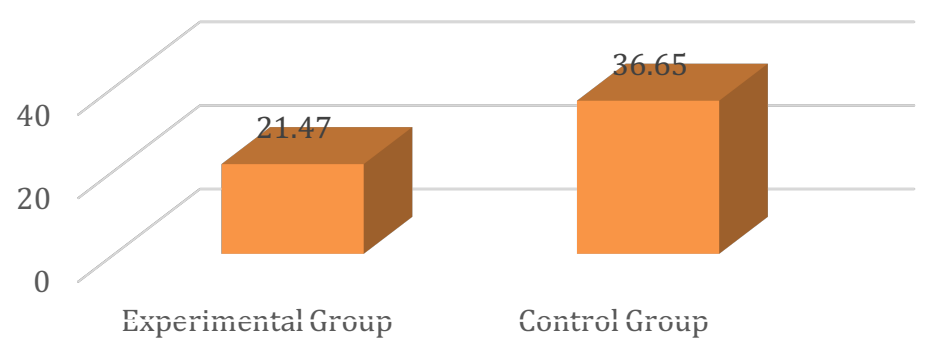

Figure 3. Comparaison de la note moyenne de groupe expérimental et de groupe de contrôl

\section{CONCLUSION}

Basée sur les notes du posttest du groupe expérimental, nous pouvons conclure que les résultats de l'apprentissage de la grammaire avec la stratégie de la classe inversée sont favorables et encourageants. Même si le nombre des étudiants ayant la note moyenne est le nombre le plus important, pourtant celui ayant la note élevée reste correct. D'ailleurs, malgré la présence d'un étudiant qui était en échec, nous pouvons conclure que c'était seulement un cas particulier, car les résultats d'analyse statistique en utilisant le Test-T ont montré 
qu'il y avait une différence significative des connaissances et performances grammaticales du groupe expérimental ayant mis en place la stratégie de la classe inversée à travers la plate-forme Edmodo, et du groupe de contrôle ayant effectué un apprentissage conventionnel dans la classe. Autrement dit, les résultats du groupe expérimental sont plus élevés de façon significative par rapport au groupe de contrôle.

Le processus d'apprentissage à travers Edmodo doit être supporté avec des matériels d'apprentissage intéressants et motivants comme l'utilisation de la vidéo. Ce processus marchait dans deux directions, cela veut dire que les apprenants pourraient poser des questions ou des commentaires sur cette plateforme. Néanmoins, l'utilisation de la plate-forme Edmodo n'est pas sans contraintes. Les apprenants indonésiens rencontrent également des obstacles, tels que de mauvaises connexions Internet, mais cela ne constitue pas un obstacle important si nous pensons à un impact positif d'Edmodo. Par conséquent, l'utilisation d'Edmodo en dehors des heures d'apprentissage est fortement recommandée pour aider les apprenants à mieux comprendre la matière en FLE.

Cette présente étude a des limitations, car elle ne traite pas des études qualitatives pouvant donner beaucoup plus d'information. Il est recommandé que la recherche ultérieure pourrait mettre en place l'observation d'apprentissage structurée afin de mieux mesurer l'efficacité de cette stratégie. D'ailleurs, la distribution d'un questionnaire ainsi que des entretiens structurés avec les apprenants sont également nécessaire à effectuer dans le cadre de connaître ses perceptions de façon compréhensible et profonde.

\section{REMERCIEMENTS}

Nous tenons à remercier tous les organismes et/ou toutes les personnes, notamment les étudiants des participants de la recherche, qui ont apporté une contribution significative à la rédaction et/ou à l'amélioration de l'article.

\section{RÉFÉRENCES}

Adewuyi, J. A., Bernard, A. O., \& Adewuyi, R. A. (2015). Problems of Learning Foreign Languages In Colleges of Education And Universities In Nigeria: A Comparative Study Of English And French Languages. International Journal Of English Language Teaching (Vol. 3). Retrieved From www.eajournals.org

Al-Harbi, S. S., \& Alshumaimeri, Y. A. (2016). The Flipped Classroom Impact in Grammar Class on EFL Saudi Secondary School Students' Performances and Attitudes. English Language Teaching. https://doi.org/10.5539/elt.v9n10p60

Al-Mekhlafi, A. M., \& Nagaratnam, R. P. (2011). Difficulties In Teaching And Learning Grammar In An Efl Context 1. International Journal (Vol. 4). Retrieved from www.e-iji.net.

Alhaysony, M., \& Alhaisoni, E. (2017). EFL Teachers' and Learners' Perceptions of Grammatical Difficulties. Advances in Language and Literary Studies, 8(1), 188-199. Retrieved from http://www.journals.aiac.org.au/inde x.php/alls/article/view/3148/2607

Beaver, J. K., Hallar, B., Westmaas, L., \& Englander, K. (2015). BLENDED LEARNING Lessons from Best Practice Sites and the Philadelphia Context PERC Research Brief. Retrieved from www.phillyeducationresearch.org.

Bergmann, J., \& Sams, A. (2012). Flip Your Classroom: Reach Every Student in Every Class Every Day. Retrieved from www.iste.org/learn/publications/per missions-and-

Butt, A. (2014). Student Views On The Use Of A Flipped Classroom Approach: Evidence From Australia. Business Education \& Accreditation, 6(1), 33-43. Retrieved from $\mathrm{ftp} / / / \mathrm{ftp}$.repec.org/opt/ReDIF/RePEc/i bf/beaccr/bea-v6n1-2014/BEA-V6N12014-4.pdf

Capone, R., De Caterina, P., \& Mazza, G. (2017). Blended Learning, Flipped Classroom and Virtual Environment: Challenges and Opportunities for the 21St Century Students. In 
EDULEARN17 Conference (pp. 1047810482).

https://doi.org/10.21125/edulearn.201 7.0985

Chachu, S. (2016). “I am speaking French but I am thinking in English": An analysis of errors by students of the French language at the University of Ghana. Ghana Journal of Linguistics, 5(1), 37-54.

Correa, M. (2015). Flipping the Foreign Language Classroom and Critical Pedagogies: A (New) Old Trend. Higher Education for the Future, 2(2), 114-125.

https://doi.org/10.1177/234763111558 4122

Creswell, J.W. (2013). Qualitative Inquiry and Research Design: Choosing Among Five Approaches. Third edition. Washington DC: Sage.

Ekmekci, E. (2017). The Flipped Writing Classroom In Turkish Efl Context: A Comparative Study On A New Model. Turkish Online Journal of Distance Education. Retrieved from http://tojde.anadolu.edu.tr/yonetim/i cerik/makaleler/1392-published.pdf

Graham, C. R. (2016). Blended learning systems: Definition, current trends, and future directions. In C. R. Bonk, C. J. \& Graham (Ed.), Handbook of blended learning: Global Perspectives, local designs. (pp. 3-21). San Francisco, CA: Pfeiffer Publishing.

Kaménova, S. (2017). Concevoir Un Cours Hybride En Français Des Affaires. Francisola, 1(2), 196. https://doi.org/10.17509/francisola.v1 i2.5557

Kara, S. (2016). How And Why? Edmodo As A Blended Learning Tool: A Brief Overview Of Usage And Research. https://doi.org/10.5171/2013.657749

Köroğlu, Z. Ç., \& Çakır, A. (2017). European Journal of English Language Teaching Use Of Flipped Instruction
In Language Classrooms; An Investigation About Student Teachers' Perceptions. European Journal of English Language Teaching, 3, 18. https://doi.org/10.5281/zenodo.85250 8

Liu, M. (2013). Blended Learning in a University EFL Writing Course: Description and Evaluation. Journal of Language Teaching and Research, $4(2)$, 301-309. https://doi.org/10.4304/jltr.4.2.301-309 Purnawarman, P., Susilawati, S., \& Sundayana, W. (2016). The use of Edmodo in teaching writing in a blended learning setting. Indonesian Journal of Applied Linguistics, 5(2), 242. https:/doi.org/10.17509/ijal.v5i2.1348

Redhouane, K., \& Gamal, P. F. B. (2017). L'Enseignement Explicite et/ ou Implicite de la Grammaire en 4ème A.M : Quelle Démarche faut-il Choisir en Classe de FLE. Revue Académique Des Études Humaines et Sociales, 18, 34-46. Retrieved from http://www.univ-

chlef.dz/ratsh/la_revue_N_18/Article _Revue_Academique_N_18_2017/Let tre_philosophie/Article_16.pdf

Shabiralyani, G., Hasan, K. S., Hamad, N., \& Iqbal, N. (2015). Impact of Visual Aids in Enhancing the Learning Process Case Research: District Dera Ghazi Khan. Journal of Education and Practice, 6(19), 226-233. Retrieved from www.iiste.org

Warden, A. (2016). Investigating the use of a Flipped Approach to Grammar Input in an English as a Foreign Language Classroom. The University of Chichester. Retrieved from https://englishagenda.britishcouncil. org/sites/default/files/attachments/m da2017_university_of_chichester_nile _alexandra_warden.pdf 